\title{
Particle creation in the early Universe: achievements and problems
}

\author{
A. A. Grib ${ }^{1,2, \dagger}$, Yu. V. Pavlov ${ }^{1,3, \ddagger}$ \\ ${ }^{1}$ A. Friedmann Laboratory for Theoretical Physics, St. Petersburg, Russia; \\ ${ }^{2}$ Theoretical Physics and Astronomy Department, The Herzen University, \\ Moika 48, St. Petersburg 191186, Russia; \\ ${ }^{3}$ Institute of Problems in Mechanical Engineering, Russian Acad. Sci., \\ Bol'shoy pr. 61, St. Petersburg 199178, Russia
}

\begin{abstract}
Results on particle creation from vacuum by the gravitational field of the expanding Friedmann Universe are presented. Finite results for the density of particles and the energy density for created particles are given for different exact solutions for different regimes of the expansion of the Universe. The results are obtained as for conformal as for nonconformal particles. The hypothesis of the origination of visible matter from the decay of created from vacuum superheavy particles identified with the dark matter is discussed.
\end{abstract}

PACS number: 04.62.+v, 98.80.Cq, 95.35.+d

Key words: particle creation, early Universe, dark matter

\section{Introduction}

The problem of particle creation from vacuum by the gravitational field of the expanding Universe was studied intensively in the 70-ies of the past century (see our works [1, 2] and papers of Ya.B. Zel'dovich and A.A. Starobinsky [3, 4,). High interest to the problem was expressed by K.P. Staniukovich [5], who always supported one of the authors of this paper (A.A.G.) in his activity. Really, differently from the usual opinion that speaking about the Big Bang one must consider very high densities of matter composed from massive particles particle creation from vacuum at the certain epoch of the evolution of the Universe makes possible to say that early Universe did not contain massive particles and looked as vacuum and possibly some fields. In works [1, 2, 6] finite values for the density of created particles and the energy density were obtained for the first time. It became clear that in the Universe described by the Friedmann metric some special era exists for every type of particles defined by the Compton time of the particle so that there is no creation of these particles for the time much smaller or much larger than this time passed from the beginning of the Universe. In papers [3, 7] an important result was obtained that in the anisotropic Universe particle creation leads to the isotropization of the metric which can be expla-

\footnotetext{
†andrei_grib@mail.ru

†yuri.pavlov@mail.ru
}

nation of the fact that our Universe is isotropic.

However after these first successes the interest to the problem somehow failed. The reason for this is the incorrect evaluation of the quantitative meaning of the effect in books of high authority [8, 9] using the hypothesis of the creation of only those particles which are observed today. Due to the fact that the effect is proportional to the mass of the particle the effect is surely negligible for small masses. However if one supposes that observable particles appeared after creation by the gravitation of massive particles with the mass of the order of the Grand Unification the result is not small at all and even more it makes possible to explain the observable number of particles in the Universe [10, 11]. The other reason of the neglect is the incorrect statement in much popular book 12 that calculations based on the method of the diagonization of the Hamiltonian lead to the infinite value of the density of the created particles for nonconformal particles. This opinion was shown to be wrong by calculations in [13. Note that in the book [12] as well as in the book [14 there are no any finite (!) values of physical evaluations of the effect of particle creation in cosmology. This occurred because of the fact that the method of the diagonalization of the Hamiltonian strongly depends on the form of the Hamiltonian used for calculation of the particle creation in Friedmann Universe. The authors of [1] were lucky in 1969 to use the correct expression of this Hamiltonian the ground state 
of which leads to the finite value of the observable quantities. This Hamiltonian is defined by the metrical energy-momentum tensor of the quantized massive field and is connected with the canonical Hamiltonian of particles with varied mass in conformally connected to the Friedmann metric static space. It is this connection which is leading to to finite results as for conformal as for nonconformal particles. Any other choice of vacuum or Hamiltonian leads to the infinite value of the density of created particles making the theory physically meaningless. In this paper after a short review of the results which can be found in our book [10] we give a review of some new results obtained by the authors in last time. Some unsolved problems are discussed in the Conclusion.

We use the system of units in which $\hbar=c=1$.

\section{Scalar field in curved Fried- mann space-time}

Consider the complex scalar field $\varphi(x)$ of the mass $m$ in curved Friedmann space-time with the Lagrangian

$$
L(x)=\sqrt{|g|}\left[g^{i k} \partial_{i} \varphi^{*} \partial_{k} \varphi-\left(m^{2}+\xi R\right) \varphi^{*} \varphi\right],
$$

leading to Euler equations

$$
\left(\nabla^{i} \nabla_{i}+\xi R+m^{2}\right) \varphi(x)=0,
$$

where $\nabla_{i}$ are covariant derivatives in metric $g_{i k}$ of the $N$-dimensional space-time, $g=\operatorname{det}\left(g_{i k}\right), R-$ the curvature scalar. For $m=0$ and $\xi=\xi_{c} \equiv(N-$ $2) /[4(N-1)]$ equation (2) is conformal invariant. This means that in mapping of the space-time with metric $g_{i k}$ on the space-time with metric $\tilde{g}_{i k}$ :

$$
g_{i k} \rightarrow \tilde{g}_{i k}=\exp [-2 \sigma(x)] g_{i k},
$$

where $\sigma(x)$ is arbitrary smooth function of coordinates there exists such scale transformation

$$
\varphi(x) \rightarrow \tilde{\varphi}(x)=\exp \left[\frac{N-2}{2} \sigma(x)\right] \varphi(x),
$$

that the wave equation conserves its form (see [10]). The physical sense of the conformal invariance is that massless field has no its proper length scale (for the massive case such a scale is given by the Compton wave length $\lambda_{C}=1 / m$ ) and so it must behave equally in conformally connected spaces (3). In case $\xi=\xi_{c}$ equation (2) is called equation with conformal coupling $\left(\xi_{c}=1 / 6\right.$ for $\left.N=4\right)$. The case $\xi=0$ corresponds to minimal coupling. Such type of coupling of the scalar field with curvature usually is supposed in inflation models [15. In quantum theory of fields in curved space-time one often considers the case of arbitrary $\xi$ [12] (more general case of coupling with the curvature of Gauss-Bonnet type was considered in [16, 17]). Then conformal invariance for massless fields is absent. The study of nonconformal case is important due to different reasons. Massive vector mesons 10 (longitudinal components) and gravitons [18 satisfy equations of this type. In case of the scalar field with self interaction in general it is impossible to conserve conformal invariance not only of the effective action (the conformal anomaly) but of the action itself [12].

Note that the Dirac equation in curved space for $m=0$ is conformal invariant without any additional modifications [10, 12.

Furthermore, let us consider an $N$-dimensional homogeneous isotropic space-time, choosing the metric in the form

$$
d s^{2}=g_{i k} d x^{i} d x^{k}=a^{2}(\eta)\left(d \eta^{2}-d l^{2}\right),
$$

where $d l^{2}=\gamma_{\alpha \beta} d x^{\alpha} d x^{\beta}$ is the metric of an $(N-1)$ dimensional space of constant curvature $K=0, \pm 1$.

The complete set of solutions to Eq. (2) in the metric (5) may be found in the form

$$
\varphi(x)=\frac{\tilde{\varphi}(x)}{a^{(N-2) / 2}(\eta)}=a^{-(N-2) / 2}(\eta) g_{\lambda}(\eta) \Phi_{J}(\mathbf{x}),
$$

where

$$
\begin{gathered}
g_{\lambda}^{\prime \prime}(\eta)+\Omega^{2}(\eta) g_{\lambda}(\eta)=0 \\
\Omega^{2}(\eta)=\left(m^{2}+\left(\xi-\xi_{c}\right) R\right) a^{2}+\lambda^{2} \\
\Delta_{N-1} \Phi_{J}(\mathbf{x})=-\left(\lambda^{2}-\left(\frac{N-2}{2}\right)^{2} K\right) \Phi_{J}(\mathbf{x})
\end{gathered}
$$

the prime denotes a derivative with respect to the conformal time $\eta$, and $J$ is the set of indices (quantum numbers) numbering the eigenfunctions of the Laplace-Beltrami operator $\Delta_{N-1}$ in $(N-1)$ dimensional space.

To perform quantization, let us expand the field $\varphi(x)$ in the complete set of solutions ([6)

$$
\varphi(x)=\int d \mu(J)\left[\varphi_{J}^{(+)} a_{J}^{(+)}+\varphi_{J}^{(-)} a_{J}^{(-)}\right],
$$

where $d \mu(J)$ is a measure on the set of quantum numbers,

$$
\varphi_{J}^{(+)}(x)=\frac{g_{\lambda}(\eta) \Phi_{J}^{*}(\mathbf{x})}{\sqrt{2} a^{(N-2) / 2}(\eta)}, \quad \varphi_{J}^{(-)}(x)=\left(\varphi_{J}^{(+)}(x)\right)^{*},
$$


and require that the standard commutation relations hold for $a_{J}^{( \pm)}$and $\stackrel{*}{a}_{J}^{( \pm)}$.

A corpuscular interpretation of a quantized field based on the method of Hamiltonian diagonalization by Bogoliubov's transformations have been proposed for the gravitational external field in paper [1].

We give the following definition of particles in the external field [10, p. 46: "In the framework of this interpretation one calls as particles (quasiparticle) creation-annihilation operators at moment $t$ those operators in terms of which the Hamiltonian of a quantized field is diagonal at the moment $t$. Therefore a quasiparticle is interpreted as an energy quantum and the measurement of the number of quasiparticles is connected with the measurement of energy. Indeed, the measurement theory in quantum mechanics requires that in the result of measuring of some physical quantity a system would found itself in a proper state of the corresponding operator. Therefore the measurement of energy inevitably transfers the system in a proper state of the Hamiltonian. To find this state the Hamiltonian must be diagonalized."

Let us build the Hamiltonian as the canonical one for the variables $\tilde{\varphi}(x)$ and $\tilde{\varphi}^{*}(x)$, for which the equation of motion does not contain their first-order derivatives with respect to the time $\eta$. Recall that the equations of motion do not change after adding a full divergence $\partial J^{i} / \partial x^{i}$ to the Lagrangian density $L(x)$. Let us choose, in the coordinate system $(\eta, \mathbf{x})$, the vector

$$
\left(J^{i}\right)=\left(\sqrt{\gamma} c \tilde{\varphi}^{*} \tilde{\varphi}(N-2) / 2,0, \ldots, 0\right),
$$

where $\gamma=\operatorname{det}\left(\gamma_{\alpha \beta}\right), c=a^{\prime} / a$. Then, using the Lagrangian density $L^{\Delta}(x)=L(x)+\partial J^{i} / \partial x^{i}$, we obtain for the momenta canonically conjugate to $\tilde{\varphi}$ and $\tilde{\varphi}^{*}$ :

$$
\pi \equiv \frac{\partial L^{\Delta}}{\partial \tilde{\varphi}^{\prime}}=\sqrt{\gamma} \tilde{\varphi}^{* \prime}, \quad \pi_{*} \equiv \frac{\partial L^{\Delta}}{\partial \tilde{\varphi}^{* \prime}}=\sqrt{\gamma} \tilde{\varphi}^{\prime},
$$

respectively. Integrating the Hamiltonian density $h(x)=\tilde{\varphi}^{\prime} \pi+\tilde{\varphi}^{* \prime} \pi_{*}-L^{\Delta}(x)$ over the hypersurface $\Sigma: \eta=$ const, we obtain the following expression for the canonical Hamiltonian:

$$
\begin{aligned}
& H(\eta)=\int_{\Sigma} d^{N-1} x \sqrt{\gamma}\left\{\tilde{\varphi}^{* \prime} \tilde{\varphi}^{\prime}+\gamma^{\alpha \beta} \partial_{\alpha} \tilde{\varphi}^{*} \partial_{\beta} \tilde{\varphi}+\right. \\
& \left.\left[\left(m^{2}+\xi R\right) a^{2}-\frac{N-2}{4}\left(2 c^{\prime}+(N-2) c^{2}\right)\right] \tilde{\varphi}^{*} \tilde{\varphi}\right\} .
\end{aligned}
$$

The Hamiltonian (14) may be written in terms of the operators $a_{J}^{( \pm)}$and $\stackrel{*}{a}_{J}^{( \pm)}$in the following way:

$$
H(\eta)=\int d \mu(J)\left[E_{J}(\eta)\left(\stackrel{*}{a}{ }_{J}^{(+)} a_{J}^{(-)}+\stackrel{*}{a}{ }_{\bar{J}}^{(-)} a_{\bar{J}}^{(+)}\right)+\right.
$$

$$
\left.+F_{J}(\eta) \stackrel{*}{a_{J}^{(+)}} a_{\bar{J}}^{(+)}+F_{J}^{*}(\eta) \stackrel{*}{a_{\bar{J}}^{(-)}} a_{J}^{(-)}\right],
$$

where

$$
E_{J}=\frac{\left|g_{\lambda}^{\prime}\right|^{2}+\Omega^{2}\left|g_{\lambda}\right|^{2}}{2}, \quad F_{J}=\frac{\vartheta_{J}}{2}\left[g_{\lambda}^{\prime 2}+\Omega^{2} g_{\lambda}^{2}\right],
$$

and we have chosen such eigenfunctions $\Phi_{J}(\mathbf{x})$ that, for arbitrary $J$, there is such $\bar{J}$ that $\Phi_{J}^{*}(\mathbf{x})=$ $\vartheta_{J} \Phi_{\bar{J}}(\mathbf{x}),\left|\vartheta_{J}\right|=1,\left(\overline{\bar{J}}=J, \vartheta_{\bar{J}}=\vartheta_{J}\right)$. Such a choice is possible due to completeness and orthonormality of the set $\Phi_{J}(\mathbf{x})$.

According to the Hamiltonian diagonalization method [10] (the nonconformal case see in [13, 19]), the functions $g_{\lambda}(\eta)$ should obey the following initial conditions:

$$
g_{\lambda}^{\prime}\left(\eta_{0}\right)=i \Omega\left(\eta_{0}\right) g_{\lambda}\left(\eta_{0}\right), \quad\left|g_{\lambda}\left(\eta_{0}\right)\right|=\Omega^{-1 / 2}\left(\eta_{0}\right) .
$$

If the quantized scalar field is in the vacuum state at the instant $\eta_{0}$, then the number density of the pairs of particles created up to the instant $\eta$ can be evaluated (for $K=0$ ) as 10 ]

$$
n(\eta)=\frac{B_{N}}{2 a^{N-1}} \int_{0}^{\infty} S_{\lambda}(\eta) \lambda^{N-2} d \lambda,
$$

where $B_{N}=\left[2^{N-3} \pi^{(N-1) / 2} \Gamma((N-1) / 2)\right]^{-1}, \quad \Gamma(z)$ is the gamma function, and

$$
S_{\lambda}(\eta)=\frac{\left|g_{\lambda}^{\prime}(\eta)-i \Omega g_{\lambda}(\eta)\right|^{2}}{4 \Omega} .
$$

As shown in [13, $S_{\lambda} \sim \lambda^{-6}$, and the integral in (18) converges for $N<7$. So the density of particles created in four dimensional Friedmann space-time is finite. Note that the first use of the method of diagonalization of the Hamiltonian in paper [20] led to the infinite density of created quasiparticles. However there not the canonical Hamiltonian for fields $\tilde{\varphi}(x)$, but the Hamiltonian which is obtained from the metrical energy momentum tensor for the scalar field with minimal coupling was used. In the work 21 created particle were defined for the so called adiabatic vacuum state in such a way that the corresponding Hamiltonian was not diagonal at any moment of time. This prevented to obtain finite results for particle creation in Friedmann space-time.

Note that the equation (7) by use of the transformation $g(\eta)=\exp z(\eta)$ is going to the following equation for the function $v(\eta) \equiv z^{\prime}(\eta)$

$$
v^{\prime}(\eta)+v^{2}(\eta)+\Omega^{2}(\eta)=0,
$$

being the Riccati equation of the general type the solutions of which cannot be expressed in finite form 
in elementary functions. So the number of scale factors $a(\eta)$ leading to exact solutions is relatively small. In cases when exact solution can be found it usually is expressed through special functions hypergeometric functions, Bessel functions etc.

\section{Particle creation in cosmological models with $p=w \varepsilon$}

The Einstein equations

$$
R_{i k}-\frac{1}{2} g_{i k} R=-8 \pi G T_{i k},
$$

with the energy-momentum tensor of the background matter $T_{k}^{i}=\operatorname{diag}(\varepsilon,-p, \ldots,-p)$ in metric (5) have the form

$$
\begin{gathered}
\frac{c^{2}+K}{a^{2}}=\frac{16 \pi G \varepsilon}{(N-1)(N-2)}, \\
-\frac{1}{a^{2}}\left[c^{\prime}+\frac{N-3}{2}\left(c^{2}+K\right)\right]=\frac{8 \pi G p}{N-2} .
\end{gathered}
$$

From (22) it follows that for $p=w \varepsilon$ where $w=$ const, the energy density of the background matter is changing according to the law $\varepsilon \sim a^{-(1+w)(N-1)}$, i.e. is decreasing with the increasing of $a$ and if $w>-1$ it is constant, for $w=-1$ it increases with increasing $a$ for $w<-1$.

For $K=0$ and $w>-(N-3) /(N-1)$ from (22) one obtains

$$
\begin{array}{r}
a=a_{0} t^{q}=a_{1} \eta^{\beta}, \quad q=\frac{2}{(N-1)(w+1)}, \\
\beta=\frac{q}{1-q}, \quad q \in(0,1) .
\end{array}
$$

Expression for the number of pairs $N_{a}(t)=n(t) a^{3}(t)$ created in the volume $a^{3}(t)$ up to the moment of time $t$ can be written as

$$
N_{a}(t)=b_{q}^{(0)}(t) \cdot\left(\frac{a\left(t_{C}\right)}{t_{C}}\right)^{N-1},
$$

where $t_{C}=1 / m$ is the Compton time. Then $b_{q}^{(0)}(t) /(1-q)^{N-1}$ is the coefficient of proportionality for the number of created particles and the number of causally disconnected regions $N_{c}(t)=$ $((1-q) a(t) / t)^{N-1}$ at the Compton time after the Big Bang.

The results of calculations [22] of the number of created particles for the scalar particles with conformal coupling don't depend on the moment of time when one puts the initial condition as well as on time of observation of created particles if these times are

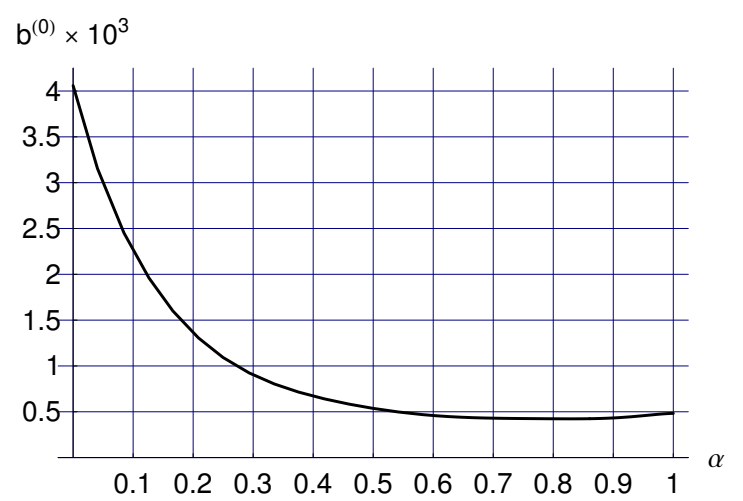

Figure 1: $b^{(0)}$ for conformal scalar particles and $a(t) \sim t^{\alpha}$.

much smaller and later than the Compton one. On Fig. 1 the results of numerical calculations of the coefficient $b^{(0)}$ for $N=4$ for different scale factors $a(t)=a_{0} t^{\alpha}$ are given. From (24) one can see the effect of connection of the number of created particles with the number of causally disconnected parts on the Friedmann Universe at the Compton time of its evolution.

The results for nonconformal scalar field can radically depend on the initial moment of time (see Eqs. (7), (8)). However for the condition $\left(\xi_{c}-\xi\right) R>$ 0 , for the case of the minimally coupled scalar field in the dust Universe $p=0\left(a(t) \sim t^{2 / 3}\right)$, the initial moment can be defined by the condition: $\Omega^{2} \geq 0$ for any $\lambda$. In the other case (negative square of energy) one can obtain change of the vacuum taking into account self interaction as it occurs for spontaneous breaking of symmetry.

Here we take for nonconformal scalar field initial value of $t_{0}$ so that $m^{2}+\left(\xi-\xi_{c}\right) R\left(t_{0}\right)=0$. The results of numerical calculations [22] of the coefficient $b^{(0)}$ for the scalar field with minimal coupling and the scale factor $a(t)=a_{0} t^{\alpha}$ are given on Fig. 2.

In the interval $q \in(0,1)$ the following two cases has the exact solutions of equations for the scalar field.

\subsection{The exact solution for $a_{0} \sqrt{t}=a_{1} \eta$}

The Friedmann model with such scale factor is very important for applications because for $K=0$ in four-dimensional space-time it corresponds to the radiationally dominated Universe. The initial conditions for the conformally coupled scalar field can be put at $\eta \rightarrow 0$ i.e. close to the singularity $a=0$

$$
\left|g_{\lambda}(0)\right|=1 / \sqrt{\lambda}, \quad g_{\lambda}^{\prime}(0)=i \lambda g_{\lambda}(0) .
$$




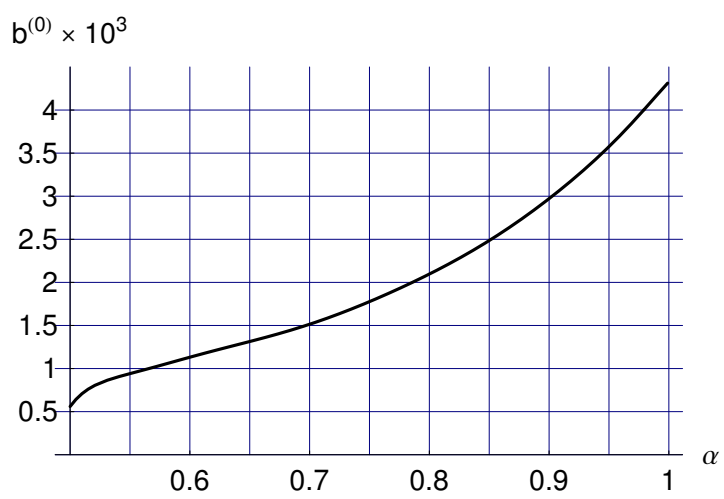

Figure 2: $b^{(0)}$ for scalar particles with minimal coupling and $a(t) \sim t^{\alpha}$.

The solution of equation (7) with conditions (25) can be written in the form

$$
\begin{array}{r}
g_{\lambda}(t)=\frac{e^{i\left(m t+\alpha_{0}\right)}}{\sqrt{\lambda}}\left[\Phi\left(\frac{1}{4}-\frac{i}{2} \delta^{2}, \frac{1}{2} ;-i 2 m t\right)+\right. \\
\left.i 2 \delta \sqrt{m t} \Phi\left(\frac{3}{4}-\frac{i}{2} \delta^{2}, \frac{3}{2} ;-i 2 m t\right)\right],
\end{array}
$$

where $\Phi(a, b ; z)$ is the degenerate hypergeometric Kummer function, $\delta \equiv \lambda /\left(m a\left(t_{C}\right)\right)$ has the sense of the physical moment at the Compton time $t_{C}=1 / \mathrm{m}$ in units $m . \alpha_{0}$ is arbitrary real constant. The representation for (26) through functions of the parabolic cylinder is given at [6]. The asymptotic value $b_{1 / 2}^{(0)} \approx$ $5,3 \cdot 10^{-4}[\underline{6}$.

\subsection{The exact solution for $a_{0} t^{1 / 3}=$ $a_{1} \sqrt{\eta}$}

The model with such scale factor for $K=0$ and $N=4$ is the Universe with limiting rigid $p=\varepsilon$ equation of state. The solution of the equation (7) with $\xi=\xi_{c}$ and initial conditions (25) for such scale factor [6]:

$$
\begin{gathered}
g_{\lambda}(t)=\frac{\pi \delta^{2} e^{i \alpha_{0}}}{\sqrt{3 \lambda}} \sqrt{(m t)^{2 / 3}+\delta^{2}} \times \\
{\left[C_{1}(\delta) J_{1 / 3}\left(\left((m t)^{2 / 3}+\delta^{2}\right)^{3 / 2}\right)+\right.} \\
\left.+C_{2}(\delta) J_{-1 / 3}\left(\left((m t)^{2 / 3}+\delta^{2}\right)^{3 / 2}\right)\right],
\end{gathered}
$$

where $J_{\nu}(x)$ - Bessel functions

$$
\begin{array}{r}
C_{1}(\delta)=J_{2 / 3}\left(\delta^{3}\right)+i J_{-1 / 3}\left(\delta^{3}\right), \\
C_{2}(\delta)=J_{-2 / 3}\left(\delta^{3}\right)-i J_{1 / 3}\left(\delta^{3}\right), \quad \delta \equiv \frac{\lambda}{m a\left(t_{C}\right)} .
\end{array}
$$

The asymptotic value for $b_{1 / 3}^{(0)} \approx 8,1 \cdot 10^{-4}$.

\subsection{The exact solution for $a(t) \sim t$}

For $w=-(N-3) /(N-1)$ one obtains from the Einstein equations (22) that $a=a_{0} t=a_{1} e^{a_{0} \eta}$. If $a_{0}=1$ and $K=-1$, then $\varepsilon=0$ and the metric (5D) with such scale factor is flat while coordinates $x^{k}$ describe the part of the Minkowski space. The four dimensional hyperbolic space-time with $a(t)=t$ is known as the Milne Universe.

In metric(5) with $a=a_{0} t$ the solution of equation (7) with $\xi=\xi_{c}$ and initial conditions (17) put at the moment $m t \rightarrow 0$ has the form

$$
g_{\lambda}(t)=\frac{\sqrt{\lambda}}{a_{0}} \Gamma\left(\frac{i \lambda}{a_{0}}\right) J_{i \lambda / a_{0}}(m t) e^{i \alpha_{0}},
$$

where $\Gamma(z)$ is the gamma function, $\alpha_{0}$ is the arbitrary real constant. Independently from $a_{0}$ and $K$ the asymptotic at $m t \gg 1$ value of the density of created quasiparticles for $N=4$ is equal to $n(t) \approx m /\left(512 \pi t^{2}\right)$. So different from zero result takes place even for the Milne Universe where gravitational field is absent and one cannot have creation of real particles! Really the space-time analysis of particle creation using correlation function of the created pair of particles introduced in 23 . shows that the corresponding correlation function of the created pair is exponentially small at distances larger than the Compton length of the particle. This means that created quasiparticles in this case are virtual pairs with the characteristic correlation length $1 / \mathrm{m}$.

\subsection{De Sitter space-time}

This is a space of constant curvature. It is a solution of Einstein equations in empty space with nonzero cosmological constant. Using the special choice of coordinates in De Sitter space one can write its metric in the form (5) with scale factor 1-3) for the De Sitter space of the first type and 4) for the De Sitter space of the second type:

$$
\begin{aligned}
& \text { 1) } a_{1} e^{H t}=\frac{-1}{H \eta}, \text { 2) } \frac{\cosh H t}{H}=\frac{1}{H \sin \eta}, \\
& \text { 3) } \frac{\sinh H t}{H}=\frac{-1}{H \sinh \eta}, \text { 4) } \frac{\cos H t}{H}=\frac{1}{H \cosh \eta} .
\end{aligned}
$$

In all these cases the equation for the scalar field (7) has the exact solution. In case 1$), t \in(-\infty,+\infty) \Leftrightarrow$ $\eta \in(-\infty, 0)$, the solution of equation (7) with conditions (17) for $\eta_{0} \rightarrow-\infty$ has the form

$$
g_{\lambda}(\eta)=\sqrt{-\frac{\pi \eta}{2}} e^{\frac{\pi}{2} \operatorname{Im} \nu} H_{\nu}^{(2)}(-\lambda \eta) e^{i \alpha_{0}},
$$




$$
\nu=\sqrt{\frac{1}{4}-\frac{m^{2}+\left(\xi-\xi_{c}\right) R}{H^{2}}},
$$

where $H_{\nu}^{(2)}(z)$ is the Hankel function, $\alpha_{0}$ is arbitrary real constant. As it was shown in 23] (and for nonconformal case for $m^{2} \geq\left(\xi_{c}-\xi\right) R$ in [24]) by the method of the space-time correlation function the created pairs must be interpreted as virtual pairs. Absence of creation of real particles in De Sitter space is confirmed by the local form of the vacuum energy-momentum tensor and zero imaginary part of the effective Lagrangian [10].

In cases $2-4$ also for the same scale factors but taking $\eta \rightarrow \gamma \eta, \gamma=$ const the solution also can be expressed through hypergeometric functions (see $\S 9.5$ in [10] and [17). For $\gamma \neq 1$ these scale factors don't describe the De Sitter space. For example the space-time with the scale factor $a(\eta)=$ $1 /(H \cosh \gamma \eta)=\sin (\gamma H t) / H$ has the evolution between two singularities.

\subsection{Particle creation in cosmology with phantom matter}

For $w<-1$ the exact solution of equation (7) exists for the value $w=-(N+1) /(N-1) \quad(w=-5 / 3$, if $N=4)$. The scale factor of metric in this case is $a=a_{0} /(-t)=a_{1} / \sqrt{-\eta}$. For $t \rightarrow-0$ there is a singularity of the Big Rip. The solution of equation (7) satisfying the conditions (17) for $\eta_{0} \rightarrow-\infty$ has the form

$$
\begin{aligned}
g_{\lambda}(\eta)=-2 i \eta \sqrt{\lambda} & \exp \left(-\frac{\pi m^{2} a_{1}^{2}}{4 \lambda}+i\left(\lambda \eta+\alpha_{0}\right)\right) \\
& \times \Psi\left(1+\frac{i m^{2} a_{1}^{2}}{2 \lambda}, 2 ;-2 i \lambda \eta\right),
\end{aligned}
$$

where $\Psi(a, b ; z)$ is the degenerate hyperbolic Tricomi function, $\alpha_{0}$ is the arbitrary real constant. For the density of particles created in $N=4$ for $t \rightarrow-0$, one obtains (look 25]) $n=m^{3} / 24 \pi^{2}$. In spite of the fact that the general number of created particles $N_{a}(t)=n(t) a^{3}(t)$ in Lagrange volume $a^{3}(t)$ is unboundedly increasing for $t \rightarrow-0$, the back reaction of particles creation and vacuum polarization of a massive, conformally coupled scalar field on the space-time metric can be neglected in the whole region where one can apply the approach of quantum field theory in curved space-time 25$]$.

\section{Exact solutions in cosmological models with $p / \varepsilon \neq$ const}

Let us make a short review of scale factors leading to exact solutions of the equation (7). Solutions for metrics

$$
a(\eta)=A+B \tanh \gamma \eta, \quad a(\eta)=\sqrt{A+B \tanh \gamma \eta},
$$

$A, B, \gamma=$ const are expressed through the hypergeometric function (see [10, $\S 9.5,[12], \S 3.4$ ).

Metric with the scale factor $a(\eta)=\sqrt{a \eta^{2}+b \eta}$ in four dimensional space-time for $\eta \ll b / a$ corresponds to the limiting rigid equation of state $p=\varepsilon$. For $\eta \gg b / a(K=0)$ this scale factor corresponds to the radiation dominated Universe $p=\varepsilon / 3$. The solution of equation (7) with $\xi=\xi_{c}$ for this scale factor can be expressed through the degenerate hypergeometric Kummer function.

Homogeneous isotropic space-time with the scale factor

$$
\begin{array}{r}
a(\eta)=a_{1} \tan \gamma \eta=a_{1} \sqrt{\exp \left(\frac{2 \gamma t}{a_{1}}\right)-1,} \\
\eta \in\left(0, \frac{\pi}{2 \gamma}\right), \quad t \in(0,+\infty)
\end{array}
$$

is describing the the radiation dominated Universe $a(t) \approx \sqrt{2 \gamma a_{1} t}$ for small times, for larger times - exponentially expanding Universe $a(t) \approx a_{1} \exp \gamma t / a_{1}$. The solution of equation (7) for the scalar field with conformal coupling and initial values at $\eta \rightarrow 0$ is expressed through the geometric function $F(a, b, c ; z)$ :

$$
\begin{array}{r}
g(\eta)=\frac{e^{i \alpha_{0}}}{\sqrt{\lambda}}(\cos \gamma \eta)\left(1+\sqrt{1-4 m^{2} a_{1}^{2} / \gamma^{2}}\right) / 2 \\
{\left[F\left(\alpha, \beta, \frac{1}{2} ; \sin ^{2} \gamma \eta\right)+i \frac{\lambda}{\gamma} \sin \gamma \eta \times\right.} \\
\left.\times F\left(\alpha+\frac{1}{2}, \beta+\frac{1}{2}, \frac{3}{2} ; \sin ^{2} \gamma \eta\right)\right], \\
\alpha, \beta=\frac{1}{4}\left[1+\sqrt{1-\frac{4 m^{2} a_{1}^{2}}{\gamma^{2}}} \pm \frac{2}{\gamma} \sqrt{\lambda^{2}-m^{2} a_{1}^{2}}\right] .
\end{array}
$$

Note that if one finds the general solution (7) for the scalar conformally coupled to the metric with the scale factor $a(\eta)$ one can obtain by redefinition $\lambda$ the general solution for the scale factor $\tilde{a}=\sqrt{a^{2}(\eta)+b^{2}}$, where $b=$ const. For example take the model with the scale factor

$$
a(\eta)=\sqrt{a_{1}^{2} \eta^{2}+b^{2}}, \quad-\infty<\eta<\infty,
$$

which in asymptotic regions $\eta \rightarrow \pm \infty$ corresponds (for $N=4$ ) to radiation dominated cosmology. The space is contracting to the minimal scale factor $a(0)=b$, is reflecting and again is expanding. 
The solution of the equation (7) with initial conditions (17) defined by the condition of the diagonalization of the Hamiltonian at the moment $\eta=0$ like (26) has the form

$$
\begin{array}{r}
g_{\lambda}(\eta)=\frac{\exp i\left(\alpha_{0}+m a_{1} \eta^{2} / 2\right)}{\left(\lambda^{2}+m^{2} b^{2}\right)^{1 / 4}} \times \\
\times\left[\Phi\left(\frac{1}{4}-\frac{i}{2} \delta^{2}, \frac{1}{2} ;-i m a_{1} \eta^{2}\right)+\right. \\
\left.+i \eta \sqrt{\lambda^{2}+m^{2} b^{2}} \Phi\left(\frac{3}{4}-\frac{i}{2} \delta^{2}, \frac{3}{2} ;-i m a_{1} \eta^{2}\right)\right],
\end{array}
$$

$\delta^{2}=\frac{\lambda^{2}+m^{2} b^{2}}{2 m a_{1}}$. The influence of the parameter $b$ on the intensity of particle creation for the case $m=1$, $a=1 / 2$ is shown on Fig. 3. It is seen that for $b \rightarrow 0$

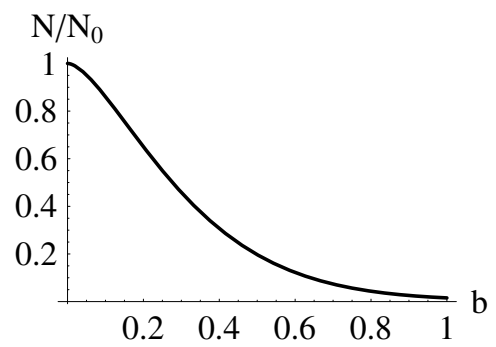

Figure 3: The relation of number of the created particles in model (36) with parameter $b$ to the radiation dominated case $(b=0)$.

the number of created particles is going to the known result for $b=0$. This shows the possibility of putting the initial conditions (17) at $b=0, \eta \rightarrow 0$, i.e. at the singularity for the radiation dominated Universe.

Note that for some scale factors the solutions for the scalar field with nonconformal coupling can be obtained by the redefinition of the mass and momentum. The problem of description of such scale factors was solved in 17 .

\section{Superheavy particles in the early Universe}

The total number of massive particles created in Friedmann radiation dominated Universe (scale factor $a(t)=a_{0} t^{1 / 2}$ ) inside the horizon is (see (24))

$$
N_{a}=n^{(s)}(t) a^{3}(t)=b^{(s)} m^{3 / 2} a_{0}^{3},
$$

where $b^{(0)} \approx 5.3 \cdot 10^{-4}$ for scalar and $b^{(1 / 2)} \approx$ $3.9 \cdot 10^{-3}$ for spinor particles 10. It occurs that $N_{a} \sim 10^{80}$ for $m \sim 10^{14} \mathrm{GeV}$ [10, 11. Calculations for creation from vacuum pairs of superheavy particles with the mass of the Grand Unification scale in the early radiation dominated Universe give the surprising result - its number is of the order of the observed Eddington-Dirac number. So putting the hypothesis of their decay on quarks and leptons in the early Universe one obtains the observed number of protons and electrons. All this shows that one cannot neglect the effect of particle creation in the early Universe!

The radiation dominance in the end of inflation era is important for our calculations. However this radiation is formed not by our visible particles. It is quintessence or some dark light particles not interacting with ordinary particles.

For the time $t \gg m^{-1}$ there is an era of going from the radiation dominated model to the dust model of superheavy particles,

$$
t_{X} \approx\left(\frac{3}{64 \pi b^{(s)}}\right)^{2}\left(\frac{M_{P l}}{m}\right)^{4} \frac{1}{m},
$$

where $M_{P l} \approx 1,2 \cdot 10^{19} \mathrm{GeV}$ is Planck mass. If $m \sim$ $10^{14} \mathrm{GeV}, t_{X} \sim 10^{-15} \mathrm{~s}$ for scalar and $t_{X} \sim 10^{-17} \mathrm{~s}$ for spinor particles.

Let us define $d$, the permitted part of long-living $X$-particles, from the condition: on the moment of recombination $t_{\text {rec }}$ in the observable Universe one has $d \varepsilon_{X}\left(t_{r e c}\right)=\varepsilon_{c r i t}\left(t_{r e c}\right)$. It leads to

$$
d=\frac{3}{64 \pi b^{(s)}}\left(\frac{M_{P l}}{m}\right)^{2} \frac{1}{\sqrt{m t_{r e c}}} .
$$

For $m=10^{13}-10^{14} \mathrm{GeV}$ one has $d \approx 10^{-12}-10^{-14}$ for scalar and $d \approx 10^{-13}-10^{-15}$ for spinor particles. So the lifetime of the main part or all $X$-particles must be smaller or equal than $t_{X}$.

We give in Ref. 26, 27, the model which can give: (a) short-living $X$-particles decay in time $\tau_{q}<t_{X}$ (more wishful is $\tau_{q} \sim t_{C} \approx 10^{-38}-10^{-35}$ s, i.e., the Compton time for $X$-particles); (b) long-living particles decay with $\tau_{l}>1 / \mathrm{m}$.

If $\tau_{l}$ is larger than the time of breaking of the Grand Unification symmetry it can be that some quantum number can be conserved leading to some effective time $\tau_{l}^{\text {eff }}>t_{U} \approx 4.3 \cdot 10^{17} \mathrm{~s}\left(t_{U}\right.$ is the age of the Universe). The small $d \sim 10^{-15}-10^{-12}$ part of long-living $X$-particles with $\tau_{l}>t_{U}$ forms the dark matter.

For $t_{l}^{\text {eff }} \leq 10^{27}$ s one must get a strong anisotropy of ultra high energy cosmic rays in the direction to the center of the Galaxy. However, experiments with cosmic rays don't show such an anisotropy and one must suppose $t_{l}^{\mathrm{eff}}>10^{27} \mathrm{~s}$. The special conditions 
existing in vicinity of black holes can lead to the collisions of superheavy particles with high energy and to their decay there on ordinary particles as it was in the early Universe. Our estimates [28] show that such processes near the black holes of active galactic nuclei can explain the stream of ultrahigh energy cosmic rays with energy $\geq 10^{19} \mathrm{eV}$ observed in experiments.

The observed entropy in this scenario originates due to transformation of $X$-particles into light particles: quarks, antiquarks and some particle similar to $\Lambda^{0}$ in $K^{0}$-meson theory, having the same quantum number as $X$. Baryon charge is created close to the time $t_{q}$, which can be equal to the Compton time of $X$-particles $t_{C} \sim 10^{-38}-10^{-35} \mathrm{~s}[22$.

\section{Conclusion}

Particle creation from vacuum by the gravitation of the expanding Friedmann Universe can explain the observable number of visible particles as well as dark matter. In our scenario it is dark matter consisting of superheavy particles with mass of the Grand Unification which is created first, ordinary particles are created not by gravitation but by the conversion of particles of dark matter into them at very high energy when high symmetry of Grand Unification is present. Breaking of this symmetry leads to conservation of some part of originally created dark matter particles which are stable today.

This hypothesis can be checked in processes with elementary particles in ergosphere of rotating black holes where collisions of particles can lead to very high energy in the centre of mass frame so the situation of the early Universe can be reproduced.

In our papers [28, 29] it was shown 1) conversion of dark matter particles with large mass into visible particles with relatively small mass can lead to observation of the products on the Earth as ultra high energy cosmic ray particles inspite of the large red shift. 2) Estimates of the process in taking into account observable dark matter density lead to observable flaws of ultra high energy cosmic rays.

There are still some unsolved problems.

a) In anisotropic space-time only in some special case [19] one can obtain the finite results by the Hamiltonian diagonalization. So this technical problem is still unsolved.

b) Quantization of minimal coupled field in general case leads to two types of modes: usual oscillations and unstable growing modes. So that one must introduce indefinite metric for their quantization. Correct quantization needs taking into account nonlinear terms (see paper 30 for some special case) leading to redefinition of vacuum as it is done in spontaneous symmetry breaking theories.

Acknowledgments. This work was supported by the Russian Foundation for Basic Research, grant No. 15-02-06818-a.

\section{References}

[1] A.A. Grib and S. G. Mamayev, Yadernaya Fizika 10, 1276-1281 (1969) [Engl. transl.: Sov. J. Nucl. Phys. (USA) 10, 722-725 (1970)]. On field theory in Friedmann space.

[2] A. A. Grib, S. G. Mamayev, and V. M. Mostepanenko, Quantum effects in intensive external fields [in Russian] (Atomizdat, Moscow, 1980).

[3] Ya. B. Zel'dovich and A. A. Starobinskii, Sov. Phys. JETP 34, 1159-1166 (1972). Particle production and vacuum polarization in an anisotropic gravitational field.

[4] Selected Works of Ya. B. Zeldovich, Vol. II: Particlies, Nuclei, and the Universe (Nauka, Moscow, 1985) [Engl. transl: Princeton Univ. Press, Princeton, 1993].

[5] K. P. Stanyukovich and V. N. Melnikov, Hydrodynamics, Fields and Constants in the Theory of Gravitation [in Russian] (Energoatomizdat, Moscow, 1983).

[6] S. G. Mamayev, V.M. Mostepanenko, and A. A. Starobinskii, Sov. Phys. JETP 43, 823-830 (1976). Particle creation from the vacuum near a homogeneous isotropic singularity.

[7] V. N. Lukash and A. A. Starobinskii, Sov. Phys. JETP 39 742-747 (1974). The isotropization of the cosmological expansion owing to particle production.

[8] Ya. B. Zel'dovich and I. D. Novikov, The Structure and Evolution of the Universe (Nauka, Moskow, 1975) [Engl. transl.: University of Chicago Press, Chicago, 1983].

[9] S. Weinberg, The First Three Minutes. A Modern View of the Origin of the Universe (Basic Books, New York, 1977).

[10] A. A. Grib, S. G. Mamayev, and V. M. Mostepanenko, Vacuum Quantum Effects in Strong Fields (Friedmann Lab. Publ., St. Petersburg, 1994). 
[11] A. A. Grib and V. Yu. Dorofeev, Int. J. Mod. Phys. D 3, 731-738 (1994). Creation of particles and entropy in the early Friedmann Universe.

[12] N.D. Birrell and P.C.W. Davies, Quantum fields in curved space (Cambridge University Press, Cambridge, 1982).

[13] Yu. V. Pavlov, Teor. Matem. Fiz. 126, 115-124 (2001) [Engl. transl.: Theor. Math. Phys. 126, 92-100 (2001)]. Nonconformal scalar field in a homogeneous isotropic space and the Hamiltonian diagonalization method.

[14] R.M. Wald, Quantum field theory in curved spacetime and black hole thermodynamics (University of Chicago Press, Chicago, 1994).

[15] A. D. Linde, Particle Physics and Inflationary Cosmology (Harwood Academic, New York, 1990).

[16] Yu. V. Pavlov, Teor. Matem. Fiz. 140, 241-255 (2004) [Engl. transl.: Theor. Math. Phys. 140, 1095-1108 (2004)]. Renormalization and dimensional regularization for a scalar field with Gauss-Bonnet-type coupling to curvature.

[17] Yu. V. Pavlov, Teor. Matem. Fiz. 174, 504-512 (2013) [Engl. transl.: Theor. Math. Phys. 174, 438-445 (2013)]. Exact solutions and particle creation for nonconformal scalar fields in homogeneous isotropic cosmological models.

[18] L.P. Grishchuk and V.M. Yudin, J. Math. Phys. 21, 1168-1175 (1980). Conformal coupling of gravitational wave field to curvature.

[19] Yu. V. Pavlov Int. J. Mod. Phys. A 17, 1041-1044 (2002). Creation of the nonconformal scalar particles in nonstationary metric.

[20] T. Imamura, Phys. Rev. 118, 1430-1434 (1960). Quantized meson field in a classical gravitational field.

[21] L. Parker. Phys. Rev. 183, 1057-1068 (1969). Quantized Fields and Particle Creation in Expanding Universe. I.

[22] A. A. Grib and Yu. V. Pavlov, Grav. Cosmol. 14, 1-7 (2008). Is dark matter the relic of the primordial matter that created the visible matter of the Universe?
[23] S. G. Mamayev and N. N. Trunov, Yadernaya Fizika 37, 1603-1612 (1983); Sov. J. Nucl. Phys. 37, 952-957 (1983). Space-time description of particle creation in gravitational and electromagnetic fields.

[24] Yu. V. Pavlov, Grav. Cosmol. 14, 314-320 (2008). Space-time description of scalar particle creation by a homogeneous isotropic gravitational field.

[25] Yu. V. Pavlov, Grav. Cosmol. 15, 341-344 (2009). On particles creation and renormalization in a cosmological model with a Big Rip.

[26] A. A. Grib and Yu. V. Pavlov, Int. J. Mod. Phys. D 11, 433-436 (2002). Superheavy particles in Friedmann cosmology and the dark matter problem.

[27] A. A. Grib and Yu. V. Pavlov, Int. J. Mod. Phys. A 17, 4435-4439 (2002). Cold dark matter and primordial superheavy particles.

[28] A. A. Grib and Yu. V. Pavlov, Mod. Phys. Lett. A 23, 1151-1159 (2008). Do active galactic nuclei convert dark matter into visible particles?

[29] A. A. Grib and Yu. V. Pavlov, Grav. Cosmol. 15, 44-48 (2009). Active galactic nuclei and transformation of dark matter into visible matter.

[30] A. A. Grib and V. Yu. Dorofeev, Grav. Cosmol. 16, 85-91(2010). Spontaneous symmetry breaking for long-wave gravitons in the early Universe. 